\title{
Intelligent Evolutionary Controller for Flexible Robotic Arm
}

\author{
Annisa Jamali ${ }^{1}$ and Intan Z. Mat Darus ${ }^{2}$ \\ ${ }^{1}$ Universiti Malaysia Sarawak, Sarawak, Malaysia \\ ${ }^{2}$ Universiti Teknologi Malaysia, Johor, Malaysia \\ Email: intan@utm.my
}

\begin{abstract}
Robotic is one of the key technologies towards Industrial Revolution 4.0. Robotic system, especially robotic arm have received tremendous demand in various fields especially manufacturing industry. Robotic arm is highly needed to enhance production, improve output, reduce human error and the most importantly, earn more profit with fast return on investment. The current industrial robotic arm, not only they are very expensive and required specialist for maintenance, they are also very heavy and difficult to manoeuvre. These facts are the reason why robotic solution are still unaffordable in most small and medium manufacturing industries in developing countries. Despite all the drawbacks, there is still a pressing need to employ robotics solution with the inherent problems of worker-related issues and output quality. Today, work requires a nimble and versatile robot and yet remain reliable. Operating robots should be simpler, where the learning curve is less steep. The user interface should be friendly and intuitive. Recently, there is a growing interest in employing lightweight, stronger and more flexible robotic arm in various fields. However, lightweight robot arm can be more easily influenced by unwanted vibrations, which may lead to problems including fatigue, instability and performance reduction. These problems may eventually cause damage to the highly stressed structures. This research focused on the development of the intelligent evolutionary controller algorithms for controlling flexible robotic arm manipulator. The controller algorithm has been formulated for trajectory planning control and vibration cancelation utilizing intelligent evolutionary algorithms such as Particle Swarm Algorithm and Artificial Bees Colony. The developed evolutionary algorithms have been implemented and experimentally verified using robotic arm manipulator experimental rig. The performances of these intelligent evolutionary controllers were found to be far better than the conventional method in term of input tracking, trajectory control and vibration cancelation.
\end{abstract}

\section{Introduction}

The research on flexible link manipulator (FLM) is reported as early as in 1970's as an alternative to solve problem portray in rigid manipulator. The former studies were initially focused on the dynamic modeling of flexible link such as lumped parameters [1], assumed mode [2]-[3] and finite elements [4]. Apart from that, researchers also investigate the dynamic modeling through system identification [5]. 
There are another group of researchers investigating and developing dynamic model with both flexible link and joint [6]-[7]. Later, some researcher even explores in difficult mathematical modeling using PDE [8]. Most of the researches described the dynamics of either single link or two link flexible manipulators. The target is to get the modeling as close as possible to the actual plant so that the controller develops for the system will be significant.

Meanwhile, the research on control development of flexible link manipulator (FLM) started in early 80 's. There are four main control areas of FLM that is end-effector position regulation, rest to rest endeffector motion in fixed time, trajectory tracking in the joint space and trajectory tracking in the operational space. A review paper written by Benosman and Le Vey [9] revealed that, the study of FLM closed loop arm is quiet shallow. Besides, most of the control development of multi-link FLM was based on open loop structure. However, to date, there are numerous researches published on these areas which cover some of the limitation addressed in previous review papers. The intensive literature on FLM can be found in the latest reviewed paper in [10] and [11]. Though there are many acceptable results accomplished, but there are still problems to be solved due to newer applications and latest technological advancement.

In recent years, the field of robotics and automation has advanced significantly driven by industrial requirements for quicker response times and lower power consumption. These demands have led the changes in robot arm design. Most of the new designs use lightweight materials and the physical configurations of a robot have been modified such that the links are longer and thinner. Flexible manipulator structure is a favorable option in current industries as it is recognized to be very efficient. In opposite to the rigid structure, it offers cost reduction, lower power consumption, improved dexterity, better maneuverability, better transportability, safer operation, light weight and lower environmental impact. However, the system produces undesirable vibration from the flexible structure. The shortcoming become severe when multi-link is involved though it provides a higher degree of freedom. Thus, the drawback received substantial attention in order to cater recent industries demand in various applications. On-going researches focused on improving the control methods in order to fulfill all the conflicting requirements.

Among available wide range controllers, PID controller is still the most widely used in the industrial environment for MIMO systems because they are often capable of providing a satisfactory performance in spite of their simple structure and intuitiveness. The main issue of PID controllers is to tune the gains. Other than that, PID controller is still significant because of its robustness performance in a wide range of operating condition and easy to implement. In this study dimension, Alam, et al. applied hybrid PDPD/ILA tune by multi-objective Genetic Algorithm optimization for SLFM [12]. Tijani, performed a multi-objective optimization using Differential Evolution (MODE) for PID controller of SLFM [13]. Another researcher has proposed an improved Bacterial Foraging Algorithms (BFA) to tune the PID controller of SLFM [14]. Bee Algorithm have been successful to optimize the hierarchical PID parameter of SLFM in [15]. Finally, PSO is used to tune one of PID parameters of the Hybrid PID-PID controller of SLFM [16]. The literatures reveal that the application of EA is limited to SLFM. However, the survey confirms that different type of EA can be used effectively in optimizing the PID controller of FLM system in various control strategy.

In this paper, a hybrid PID-PID controller is developed for DLFRM. The global search PSO and ABC are utilized to optimize all the PID controllers' gains. The dynamic model of the system is developed through system identification using Neural Network. NARX model structure based on multi-layer perceptron is employed to obtain the non-parametric modeling in comparison to Elman neural networks [17]. The control structure of PID controllers optimize by PSO and ABC are proposed for position tracking and end point vibration suppression. Performances of the proposed controllers are implemented through simulation in MATLAB/Simulink environment. 